\title{
Luminescence and Scintillation Properties of LiF:W Single Crystal for Thermal-Neutron Detection
}

\author{
Noriaki Kawaguchi, Naoki Kawano, Go Okada, and Takayuki Yanagida \\ Nara Institute of Science and Technology 8916-5 Takayama, Ikoma, Nara 630-0192, Japan
}

(Received April 1, 2017; accepted August 21, 2017)

Keywords: neutron scintillator, luminesce, scintillation, single crystal, fluoride

The luminescence and scintillation properties of LiF:W were investigated in comparison with those of $\mathrm{LiCaAlF}_{6}$ :Eu and GS20 (Ce-doped lithium silicate glass). LiF:W showed photoluminescence and scintillation emission peaking at the wavelength of $440 \mathrm{~nm}$ with the decay time of approximately $43 \mu \mathrm{s}$, which was shorter than those of $\mathrm{LiCaAlF}_{6}: \mathrm{Eu}$ and GS20. The light yield of LiF:W estimated by pulse height spectroscopy was approximately 90 photons/neutron.

\section{Introduction}

Inorganic phosphors are used for detecting ionizing radiations such as X-rays, $\gamma$-rays, and neutrons. ${ }^{(1)}$ They convert radiation to light so that radiation can be indirectly measured by using conventional photodetectors. The conversion mechanisms vary depending on the phosphor types and applications. For example, storage phosphors (known as thermally stimulated luminescence, ${ }^{(2)}$ optically stimulated luminescence, ${ }^{(3-6)}$ and radio-photoluminescence ${ }^{(7-11)}$ ) are used to record information on radiation dose and distribution in 2- and 3-dimensional scales whereas scintillators immediately convert radiation to light, so they are advantageous for online measurements. ${ }^{(12)}$ In recent years, such phosphor materials in particular are of considerable interest as an alternative to neutron detectors using ${ }^{3} \mathrm{He}$ gas, which is, in fact, suffering from a severely rapid decrease in supply but is still being relied on as the most common detection element used in thermal-neutron detectors. ${ }^{(13,14)}$ In scintillation counters that consist of a solid thermal-neutron scintillator and a photodetector, for example, the scintillator can contain ${ }^{6} \mathrm{Li}$ elements so that they convert thermal neutrons into high-energy charged particles, and these charged particles excite luminescence centers in the scintillator material. Hence, thermal-neutron scintillators can emit luminescence in response to the incident thermal neutrons. The main required characteristics are high light yield, fast decay time, adequate emission spectrum detectable using typical photodetectors, and high ${ }^{6} \mathrm{Li}$ density (or high interaction probabilities). The details of the required characteristics and properties of traditional materials were reviewed by van Eijk et al., ${ }^{(15)}$ who comprehensively covered, for example, $\mathrm{LiF} / \mathrm{ZnS}: \mathrm{Ag}$, LiI:Eu, $\mathrm{Cs}_{2} \mathrm{LiYCl}_{6}: \mathrm{Ce}$, and GS20 (Ce-doped lithium silicate glass). The $\mathrm{LiF} / \mathrm{ZnS}: \mathrm{Ag}$ scintillator is a mixture of $\mathrm{LiF}$ and $\mathrm{ZnS}: \mathrm{Ag}$ and appears to be opaque owing to the difference in the refractive indices, so it is difficult to apply to photon-counting uses. The LiI:Eu scintillator and the $\mathrm{Cs}_{2} \mathrm{LiYCl}_{6}$ :Ce scintillator show high light yields under thermalneutron irradiation (50000 and 70000 photons/neutron, respectively); however, both materials show

${ }^{*}$ Corresponding author: e-mail: n-kawaguchi@ms.naist.jp

http://dx.doi.org/10.18494/SAM.2017.1623 
a large degree of hygroscopicity that limits their application. The GS20 glass scintillator is nonhygroscopic and shows an acceptable light yield ( 6000 photons/neutron). In recent years, we have developed $\mathrm{LiCaAlF}_{6}: \mathrm{Eu}{ }^{(16-20)}$ which shows nonhygroscopicity and higher light yield (typically $\sim 20000$ photons/neutron) than that of GS20.

We describe the characteristics of a $\mathrm{LiF}: \mathrm{W}$ ( $\mathrm{LiF}$ doped with $\mathrm{WO}_{3}$ ) single crystal as a thermal-neutron scintillator. The LiF single crystal is an attractive host material because of its high ${ }^{6} \mathrm{Li}$ density. According to the literature, ${ }^{(21)}$ the ${ }^{6} \mathrm{Li}$ density of GS20 is approximately $2.2 \times 10^{22}$ atoms $/ \mathrm{cm}^{3}$. In addition, ${ }^{6} \mathrm{Li}$ densities of ${ }^{6} \mathrm{Li}$-enriched $(95 \%) \mathrm{LiCaAlF}_{6}, \mathrm{LiI}$, and $\mathrm{LiF}$ are approximately $0.91 \times 10^{22}, 1.8 \times 10^{22}$, and $5.8 \times 10^{22}$ atoms $/ \mathrm{cm}^{3}$, respectively. Therefore, LiFbased materials are potentially applicable for high-efficiency thermal-neutron detection. The LiF single crystal is difficult to dope with typical efficient luminescent centers (e.g., rare-earth ions). Only some oxide compounds (e.g., $\mathrm{WO}_{3}, \mathrm{TiO}_{2}$, and $\mathrm{Nb}_{2} \mathrm{O}_{5}$ ) are known as possible dopants. ${ }^{(22-24)}$ In particular, the luminescence of the LiF:W single crystal is more intense than those doped with other oxide compounds. ${ }^{(22)}$ The origin of the luminescence in LiF:W has been investigated and ascribed to the $\mathrm{W}-\mathrm{O}$ emission complex. ${ }^{(25)}$ Other groups have pointed out the possibility of using LiF:W as a thermal-neutron scintillator; ${ }^{(26,27)}$ however, detailed scintillation properties have not yet been quantitatively reported. In the present study, we tested the LiF:W single crystal as a thermal-neutron scintillator, and the scintillation characteristics were compared with those of other conventional neutron scintillators.

\section{Materials and Methods}

Table 1 shows the specifications of the tested materials. The GS20 glass scintillator, the $\mathrm{LiCaAlF}_{6}: \mathrm{Eu}$ single crystal, and the LiF:W single crystal were provided by Saint-Gobain S.A., Tokuyama Corp., and Ohyo Koken Kogyo Co., Ltd., respectively. All the samples showed no hygroscopicity and were air-stable at room temperature.

Photoluminescence (PL) contour maps and PL quantum yields were obtained using Hamamatsu Quantaurus-QY C11347-01 (excitation wavelength 250-400 nm, emission wavelength 200-950 $\mathrm{nm}$ ). X-ray excited luminescence spectra were measured by using an X-ray generator (Tungsten target, $80 \mathrm{kV}, 2.5 \mathrm{~mA}$; XRB80P\&N200X4550, Spellman) and CCD spectrometer (DU-920-BU2NC, Andor). The details of the setup can be found elsewhere. ${ }^{(28)}$

Scintillation decay curves were characterized by both neutron and X-ray irradiation. For the neutron-induced scintillation, the decay curves were measured using a photomultiplier tube (PMT; R7600U-200, Hamamatsu Photonics K.K.) and a digital oscilloscope (TDS 3052C, Tektronix) in a single shot mode during the irradiation of thermal neutrons by a moderated ${ }^{252} \mathrm{Cf}$ sealed radiation source. The X-ray-induced scintillation decay curve of LiF:W was obtained with a pulsed X-ray

Table 1

Specifications of tested materials.

\begin{tabular}{|c|c|c|c|}
\hline Material & Supplier & Dimensions ${ }^{*}$ & Type \\
\hline $\begin{array}{l}\text { GS20 } \\
\text { (Ce-doped lithium silicate glass) }\end{array}$ & Saint-Gobain S.A. & $10 \times 10 \mathrm{~mm}^{2}, t=1 \mathrm{~mm}$ & Glass \\
\hline $\mathrm{LiCaAlF}_{6}: \mathrm{Eu}$ & Tokuyama Corp. & $10 \times 10 \mathrm{~mm}^{2}, t=1 \mathrm{~mm}$ & Single crystal \\
\hline LiF:W & Ohyo Koken Kogyo Co., Ltd. & $\phi^{*}=25.4 \mathrm{~mm}, t=25.4 \mathrm{~mm}$ & Single crystal \\
\hline
\end{tabular}


source operated together with a time-correlated single-photon counting system (Hamamatsu Photonics K.K.). ${ }^{(29)}$ The obtained decay curves were fitted by

$$
I(t)=I(0) \exp (-t / \tau)+C
$$

where $I(t)$ is the luminescence intensity as a function of time $t, I(0)$ is the luminescence intensity at $t=0$, $\tau$ is the scintillation decay time, and $C$ is a constant.

Scintillation light yields under thermal-neutron and $\gamma$-ray irradiation were measured using a PMT (R7600U-200, Hamamatsu Photonics K.K.). A scintillator sample was optically coupled with the PMT via a silicone grease. A moderated ${ }^{252} \mathrm{Cf}$ sealed source was used as the thermal neutron source. ${ }^{22} \mathrm{Na}$ and ${ }^{137} \mathrm{Cs}$ were used as $\gamma$-ray sources. Pulse height spectra were obtained by processing the output signal from the PMT using a preamplifier (113 Scintillation Preamplifier, ORTEC), a shaping amplifier (CP4479, CLEAR-PULSE), and a multichannel analyzer (MCA 8000A, Amptek). The shaping time chosen was $20 \mu$ s for LiF:W and $5 \mu$ s for GS20 and $\mathrm{LiCaAlF}_{6}$ for both thermal-neutron and $\gamma$-ray characterizations.

\section{Results and Discussion}

Figure 1 shows the LiF:W single crystal under ambient light and $254 \mathrm{~nm}$ UV light. The LiF:W single crystal is highly transparent under ambient light and emits blue luminescence under UV light. Figure 2 shows PL contour maps (luminescent intensity versus excitation and emission wavelengths) of $\mathrm{LiF}: \mathrm{W}, \mathrm{LiCaAlF}_{6}: \mathrm{Eu}$, and GS20. LiF:W shows a broad luminescence in the wavelength range from 350 to $600 \mathrm{~nm}$ with a peak at $440 \mathrm{~nm}$ during excitation at 250 to $280 \mathrm{~nm}$. In contrast, $\mathrm{LiCaAlF}_{6}$ : $\mathrm{Eu}$ and GS20 show emissions peaking at 370 and $395 \mathrm{~nm}$, respectively, while the excitation bands are in the range of $250-350 \mathrm{~nm}$. These luminescence features are consistent with previous reports. ${ }^{(15,17)}$ All the samples showed emission in the spectral range detectable by typical PMTs. The PL quantum yields of $\mathrm{LiF}: \mathrm{W}, \mathrm{LiCaAlF}_{6}: \mathrm{Eu}$, and GS20 were approximately $26 \%\left(\lambda_{e x}=250 \mathrm{~nm}\right), 100 \%\left(\lambda_{e x}=320 \mathrm{~nm}\right)$, and $80 \%\left(\lambda_{e x}=340 \mathrm{~nm}\right)$, respectively. The PL quantum yield of LiF:W was significantly lower than those of $\mathrm{LiCaAlF}_{6}: \mathrm{Eu}$ and GS20. Figure 3 shows the X-ray-induced radioluminescence spectra of $\mathrm{LiF}_{\mathrm{W}}, \mathrm{LiCaAlF}_{6}: \mathrm{Eu}$, and GS20. The emission peaks of $\mathrm{LiF}: \mathrm{W}, \mathrm{LiCaAlF}_{6}: \mathrm{Eu}$, and GS20 were observed at 440, 370, and $395 \mathrm{~nm}$, respectively, consistent with those of PL. The radioluminescence intensity of $\mathrm{LiCaAlF}_{6}: \mathrm{Eu}$ was the highest and that of

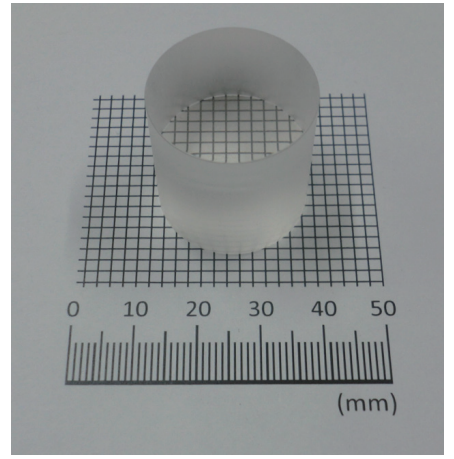

(a)

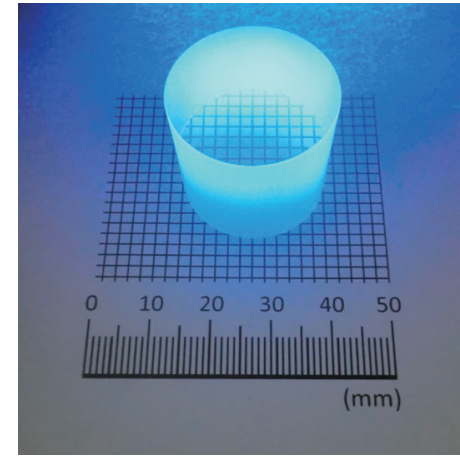

(b)

Fig. 1. (Color online) LiF:W single crystal under (a) ambient and (b) $254 \mathrm{~nm}$ UV light. 


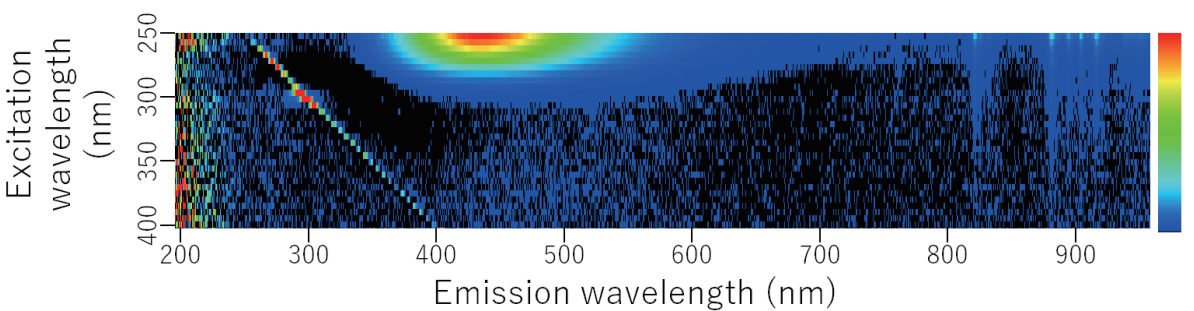

(a)

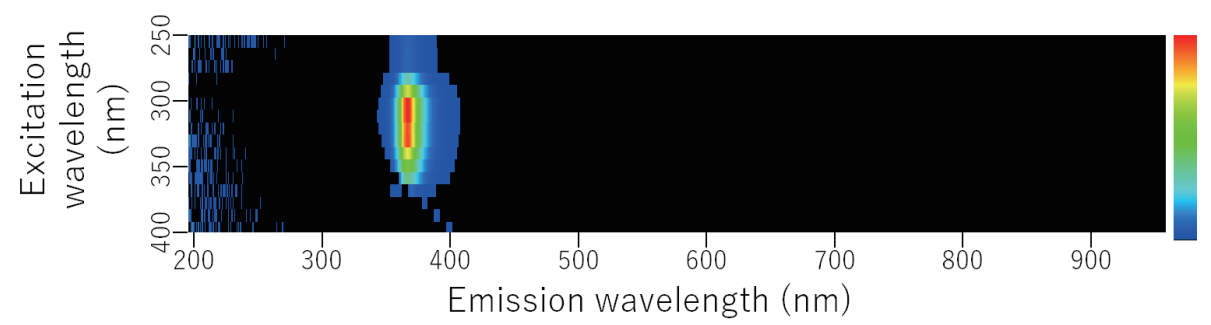

(b)

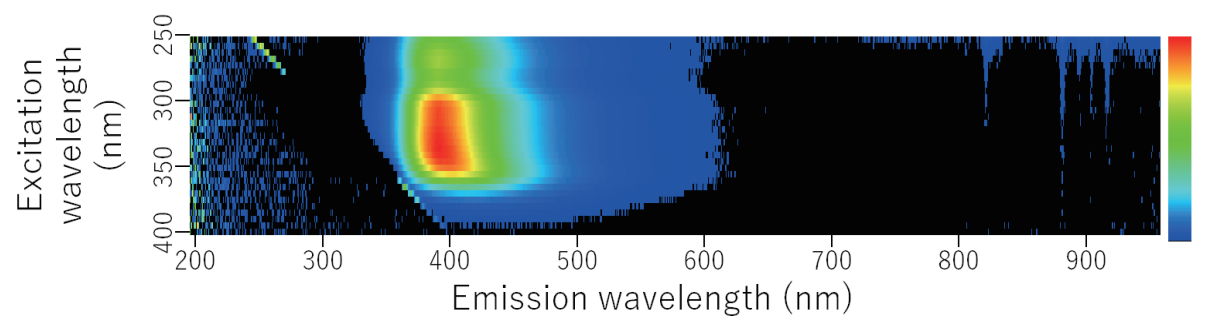

(c)

Fig. 2. (Color online) PL contour maps (luminescent intensity vs excitation and emission wavelengths) of (a) $\mathrm{LiF}: \mathrm{W}$, (b) $\mathrm{LiCaAlF}_{6}: \mathrm{Eu}$, and (c) GS20.

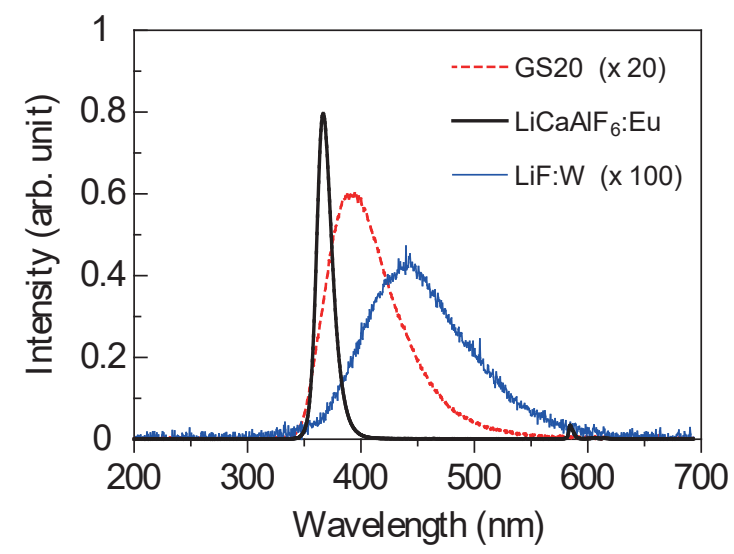

Fig. 3. (Color online) Scintillation spectra of $\mathrm{GS}_{20}, \mathrm{LiCaAlF}_{6}: \mathrm{Eu}$, and $\mathrm{LiF}: \mathrm{W}$ under X-ray irradiation. 
LiF:W was the lowest among the present samples. The correlation in fact follows the order of PL quantum yields. Although the radioluminescence intensity depends on not only the light yield but also the X-ray absorption efficiency, the radioluminescence intensities of these samples seem to be strongly affected by the PL quantum yields.

Figure 4 shows thermal-neutron-induced scintillation decay curves. Despite the low signalto-noise ratio, adequate decay curves were observed for GS20 and $\mathrm{LiCaAlF}_{6}$ :Eu. The reported decay constants are $75 \mathrm{~ns}$ and $1.6 \mathrm{~ms}$, respectively, ${ }^{(15,17)}$ and our measurements reproduced those results. In the case of LiF:W, a clear decay curve under thermal-neutron irradiation was not obtained owing to the low luminescence intensity; therefore, the decay profile was characterized by X-ray irradiation. ${ }^{(29)}$ Figure 5 shows the X-ray-induced decay curve of LiF:W. A clear decay curve under X-ray irradiation was successfully obtained. The obtained decay curve was fitted to a single-exponential function, and the obtained parameters were $I(0)=3903$ and $\tau=43.25 \mathrm{~ms}$. The estimated decay time was significantly longer than those of $\mathrm{LiCaAlF}_{6}$ : $\mathrm{Eu}$ and GS20.

Figure 6 shows pulse height spectra of LiF:W measured under thermal-neutron and $\gamma$-ray irradiation. A thermal-neutron detection peak was successfully obtained with LiF:W while only events due to Compton scattering were observed under ${ }^{137} \mathrm{Cs}$ and ${ }^{22} \mathrm{Na} \gamma$-ray irradiation owing to the low effective atomic number and low density. The pulse height of the scintillation from LiF:W under thermal-neutron irradiation was lower than those of the events of Compton scattering by $1.275 \mathrm{MeV} \gamma$-rays from ${ }^{22} \mathrm{Na}$, so the $\mathrm{n} / \mathrm{g}$ discrimination capability of LiF:W was insufficient. Figure 7 shows the pulse height spectra of LiF:W, GS20, and $\mathrm{LiCaAlF}_{6}:$ Eu under thermalneutron irradiation. All the samples showed clear peaks corresponding to the events of thermalneutron detection. The peak positions of LiF:W, GS20, and $\mathrm{LiCaAlF}_{6}: \mathrm{Eu}$ were 29, 2177, and 8134 channels, respectively. Taking into account the quantum efficiency of the PMT (R7600U-200; $42.09,41.01$, and $36.07 \%$ at 370,400 , and $440 \mathrm{~nm}$, respectively) and the known light yield of GS20 ( 6000 photons/neutron ${ }^{(15)}$ ), the light yields of $\mathrm{LiF}_{\mathrm{W}}$ and $\mathrm{LiCaAlF}_{6}: \mathrm{Eu}$ were estimated to be approximately 90 and 20000 photons/neutron, respectively. The light yield of LiF:W was found to be significantly lower than those of $\mathrm{LiCaAlF}_{6}: \mathrm{Eu}$ and GS20; however, the LiF single crystal is still an attractive host material for thermal-neutron detection because of the high ${ }^{6} \mathrm{Li}$ density, so it still remains a possibility that LiF-based materials may be used in practical applications if the light yield is somewhat improved. For further improvements, other possible luminescent centers such as defects or other dopants should be considered.

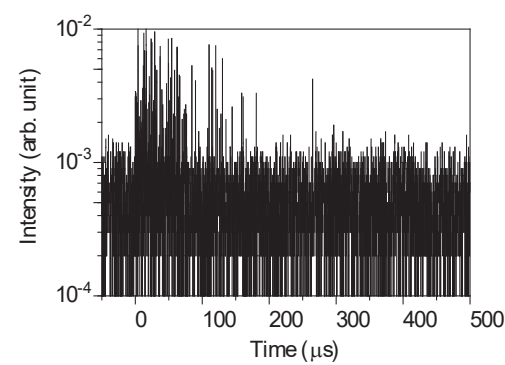

(a)

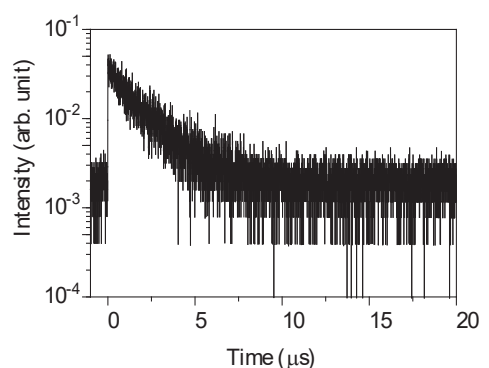

(b)

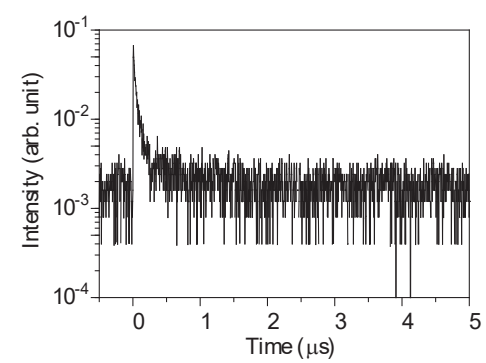

(c)

Fig. 4. Scintillation decay curves of (a) LiF:W, (b) $\mathrm{LiCaAlF}_{6}: \mathrm{Eu}$, and (c) GS20 under thermal-neutron irradiation. 


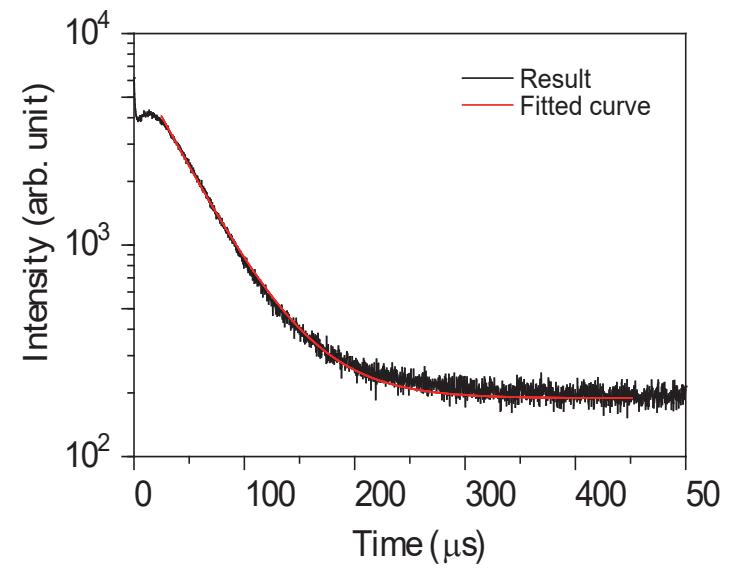

Fig. 5. (Color online) Scintillation decay curve of LiF:W under X-ray irradiation.

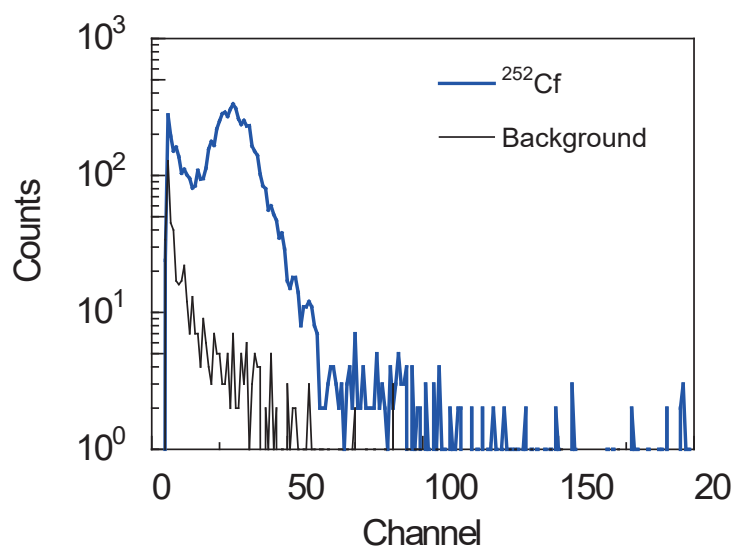

(a)

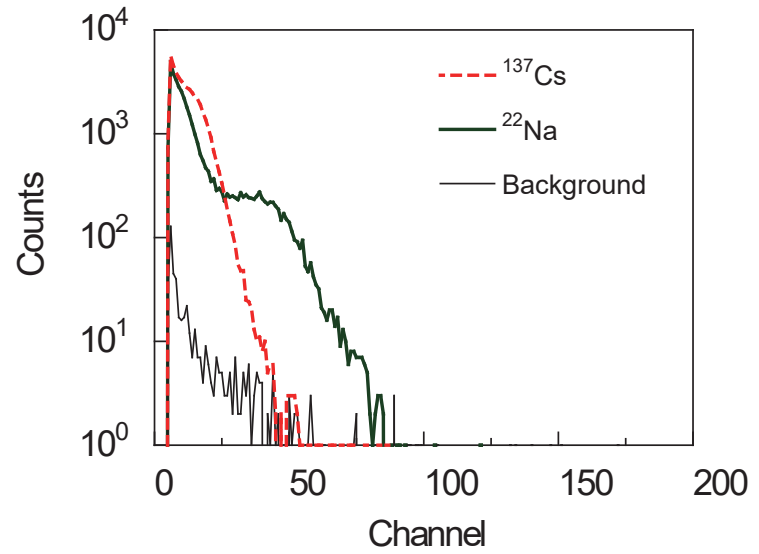

(b)

Fig. 6. (Color online) Pulse height spectra of LiF:W under (a) thermal-neutron and (b) $\gamma$-ray irradiation.

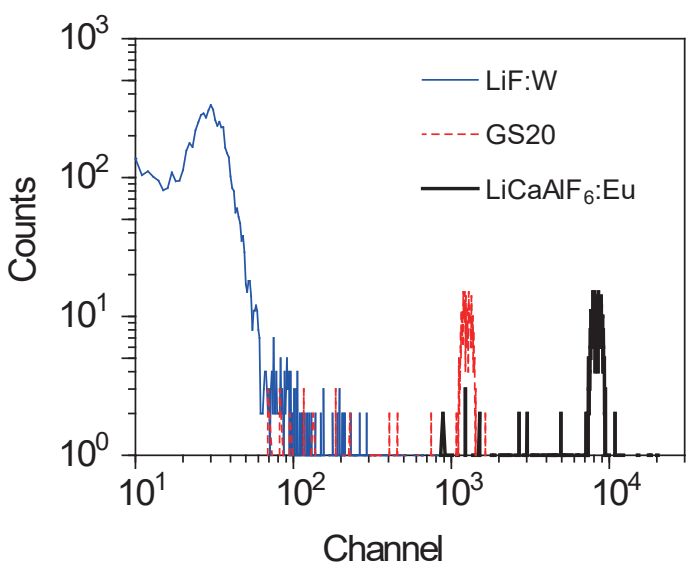

Fig. 7. (Color online) Pulse height spectra of LiF:W, GS20, and $\mathrm{LiCaAlF}_{6}$ :Eu under thermal-neutron irradiation. 


\section{Conclusions}

The LiF single crystal as a host material of the thermal-neutron scintillator is particularly interesting because of the high ${ }^{6} \mathrm{Li}$ density $\left(95 \%{ }^{-}{ }^{6} \mathrm{Li}\right.$-enriched $\mathrm{LiF}$ has approximately $5.8 \times 10^{22}$ atoms $/ \mathrm{cm}^{3}$ ), and we showed the capabilities of the LiF:W single crystal in the respective applications by comparison with the properties of the $\mathrm{LiCaAlF}_{6}:$ Eu single crystal and GS20. LiF:W showed a luminescence peak at around $440 \mathrm{~nm}$ for both PL and scintillation, and the decay time was approximately $43 \mu \mathrm{s}$, which is considerably longer than those of $\mathrm{LiCaAlF}_{6}: \mathrm{Eu}$ and Li-glass (GS20). Despite the low PL quantum yield, a thermal-neutron detection peak was successfully obtained from the pulse height spectrum using LiF:W under thermal-neutron irradiation. The light yield of LiF:W was estimated to be approximately 90 photons/neutron by comparing with GS20. The light yield should be improved for practical applications; however, LiF-based materials still remain a class of potential thermal-neutron scintillator materials owing to their high interaction probabilities.

\section{Acknowledgments}

This work was supported by a Grant-in-Aid for Scientific Research (A)-26249147 and a Grantsin-Aid for Research Activity Start-up (16H06983 and 15H06409) from the Ministry of Education, Culture, Sports, Science and Technology (MEXT) of the Japanese government, as well as by the A-STEP and Matching Planner Program of the Japan Science and Technology Agency (JST). The Cooperative Research Project of the Research Institute of Electronics, Shizuoka University, KRF Foundation, Murata Science Foundation, Hitachi Metals Materials Science Foundation, and Inamori Foundation are also acknowledged.

\section{References}

1 G. F. Knoll: Radiation Detection and Measurement, 4th ed. (Wiley, New York, 2010).

2 S. W. S. McKeever: Thermoluminescence of Solids (Cambridge University Press, Cambridge, 1985).

3 E. G. Yukihara and S. W. S. McKeever: Optically Stimulated Luminescence (Wiley, Chichester, UK, 2011).

4 S. W. S. McKeever: Nucl. Instrum. Methods Phys. Res., Sect. B 184 (2001) 29.

5 G. Okada, K. Fukuda, S. Kasap, and T. Yanagida: Photonics 3 (2016) 23.

6 N. M. Winch, A. Edgar, and C. M. Bartle: Nucl. Instrum. Methods Phys. Res., Sect. A 763 (2014) 394.

7 H. Nanto, Y. Miyamoto, T. Oono, Y. Takei, T. Kurobori, and T. Yamamoto: Procedia Eng. 25 (2011) 231.

8 G. Okada, B. Morrell, C. Koughia, A. Edgar, C. Varoy, G. Belev, T. Wysokinski, D. Chapman, and S. Kasap: Appl. Phys. Lett. 99 (2011) 121105.

9 G. Okada, J. Ueda, S. Tanabe, G. Belev, T. Wysokinski, D. Chapman, D. Tonchev, and S. Kasap: J. Am. Ceram. Soc. 97 (2014) 2147.

10 Y. Miyamoto, H. Nanto, T. Kurobori, Y. Fujimoto, T. Yanagida, J. Ueda, S. Tanabe, and T. Yamamoto: Radiat. Meas. 71 (2014) 529.

11 G. Okada, Y. Fujimoto, H. Tanaka, S. Kasap, and T. Yanagida: J. Rare Earths 34 (2016) 769.

12 T. Yanagida: Opt. Mater. 35 (2013) 1987.

13 R. T. Kouzes: Pacific Northwest National Laboratory Publications PNNL-18388 (2009).

14 R. T. Kouzes, J. H. Ely, L. E. Erikson, W. J. Kernan, A. T. Lintereur, E. R. Siciliano, D. L. Stephens, D. C. Stromswold, R. M. Van Ginhoven, and M. L. Woodring: Nucl. Instrum. Methods Phys. Res., Sect. A 623 (2010) 1035.

15 C. W. E. van Eijk, A. Bessière, and P. Dorenbos: Nucl. Instrum. Methods Phys. Res., Sect. A 529 (2004) 260.

16 N. Kawaguchi, T. Yanagida, A. Novoselov, K. J. Kim, K. Fukuda, A. Yoshikawa, M. Miyake, and M. Baba: Proc. Nuclear Science Symp., ed. P. Sellin (IEEE, Dresden, 2008) pp. 1174-1176. 
17 T. Yanagida, N. Kawaguchi, Y. Fujimoto, K. Fukuda, Y. Yokota, A. Yamazaki, K. Watanabe, J. Pejchal, A. Uritani, T. Iguchi, and A. Yoshikawa: Opt. Mater. 33 (2011) 1243.

18 T. Yanagida, A. Yamaji, N. Kawaguchi, Y. Fujimoto, K. Fukuda, S. Kurosawa, A. Yamazaki, K. Watanabe, Y. Futami, Y. Yokota, A. Uritani, T. Iguchi, A. Yoshikawa, and M. Nikl: Appl. Phys. Express 4 (2011) 106401.

19 G. Okada, K. Fukuda, N. Kawaguchi, and T. Yanagida: Radiat. Meas. (in press).

20 T. Yanagida: J. Lumin. 169 (2016) 544.

21 G. Ban, K. Bodek, T. Lefort, O. Naviliat-Cuncic, E. Pierre, C. Plonka, and G. Rogel: Nucl. Instrum. Methods Phys. Res., Sect. A 611 (2009) 280.

22 V. Taranyuk, A. Gektin, N. Shiran, V. Shlyakhturov, S. Gridin, I. Boiaryntseva, and D. Sofronov: J. Cryst. Growth 380 (2013) 205.

23 L. A. Lisitsyna, V. I. Korepanov, A. A. Abdrakhmetova, N. N. Timoshenko, and A. K. Dauletbekova: Opt. Spectrosc. 112 (2012) 175.

24 A. Dauletbekova, L. Lisitsyna, V. Korepanov, V. Lisitsyn, L. Trefilova, and R. Kassymkanova: Phys. Status Solidi C 10 (2013) 263.

25 L. A. Lisitsyna, I. A. Tupitsyna, and L. N. Trefilova: IOP Conf. Ser.: Mater. Sci. Eng. 81 (2015) 012024.

26 B. Pritychenko, A. Da Silva, A. Smith, P. D. Barnes, and B. Sadoulet: Nucl. Instrum. Methods Phys. Res., Sect. A 396 (1997) 371.

27 R Nowotny: Phys. Med. Biol. 49 (2004) 2599.

28 T. Yanagida, K. Kamada, Y. Fujimoto, H. Yagi, and T. Yanagitani: Opt. Mater. 35 (2013) 2480.

29 T. Yanagida, Y. Fujimoto, T. Ito, K. Uchiyama, and K. Mori: Appl. Phys. Express. 7 (2014) 062401. 